\title{
Combination of Antifungal Drugs and Protease Inhibitors Prevent Candida albicans Biofilm Formation and Disrupt Mature Biofilms
}

\author{
Matthew B. Lohse ${ }^{1,2}$, Megha Gulati ${ }^{4}$, Charles S. Craik ${ }^{4}$, Alexander D. Johnson ${ }^{1 *}$ and \\ Clarissa J. Nobile ${ }^{3 *}$ \\ ${ }^{1}$ Department of Microbiology and Immunology, University of California, San Francisco, San Francisco, CA, United States, \\ ${ }^{2}$ Department of Biology, BioSynesis, Inc., San Francisco, CA, United States, ${ }^{3}$ Department of Molecular and Cell Biology, \\ University of California, Merced, Merced, CA, United States, ${ }^{4}$ Department of Pharmaceutical Chemistry, University of \\ California, San Francisco, San Francisco, CA, United States
}

OPEN ACCESS

Edited by:

Juliana Campos Junqueira, São Paulo State University, Brazil

Reviewed by: Rohitashw Kumar University at Buffalo, United States Isabel M. Miranda, University of Porto, Portugal

${ }^{*}$ Correspondence: Alexander D. Johnson ajohnson@cgl.ucsf.edu

Clarissa J. Nobile

cnobile@ucmerced.edu

${ }^{\dagger}$ Present address: Megha Gulati, Molecular Cell - Cell Press, Cambridge, MA, United States

Specialty section: This article was submitted to

Infectious Diseases,

a section of the journal

Frontiers in Microbiology

Received: 11 March 2020 Accepted: 27 April 2020

Published: 25 May 2020

Citation: Lohse MB, Gulati M, Craik CS, Johnson AD and Nobile CJ (2020) Combination of Antifungal Drugs and Protease Inhibitors Prevent Candida albicans Biofilm Formation

and Disrupt Mature Biofilms.

Front. Microbiol. 11:1027.

doi: 10.3389/fmicb.2020.01027
Biofilms formed by the fungal pathogen Candida albicans are resistant to many of the antifungal agents commonly used in the clinic. Previous reports suggest that protease inhibitors, specifically inhibitors of aspartyl proteases, could be effective antibiofilm agents. We screened three protease inhibitor libraries, containing a total of 80 compounds for the abilities to prevent $C$. albicans biofilm formation and to disrupt mature biofilms. The compounds were screened individually and in the presence of subinhibitory concentrations of the most commonly prescribed antifungal agents for Candida infections: fluconazole, amphotericin B, or caspofungin. Although few of the compounds affected biofilms on their own, seven aspartyl protease inhibitors inhibited biofilm formation when combined with amphotericin B or caspofungin. Furthermore, nine aspartyl protease inhibitors disrupted mature biofilms when combined with caspofungin. These results suggest that the combination of standard antifungal agents together with specific protease inhibitors may be useful in the prevention and treatment of $C$. albicans biofilm infections.

Keywords: Candida albicans, biofilms, antimicrobial resistance, therapeutics, protease inhibitors, aspartyl protease inhibitors

\section{INTRODUCTION}

Candida albicans is a member of the human microbiota which asymptomatically colonizes the skin, mouth, and gastrointestinal tract of healthy humans (Douglas, 2003; Nobile and Johnson, 2015; Gulati and Nobile, 2016; Lohse et al., 2018). This fungal species is also one of the most common pathogens of humans, typically causing superficial dermal and mucosal infections (Kennedy and Volz, 1985; Kullberg and Oude Lashof, 2002; Kumamoto, 2002, 2011; Douglas, 2003; Achkar and Fries, 2010; Ganguly and Mitchell, 2011; Kim and Sudbery, 2011). When a host's immune system is compromised (e.g., in patients undergoing chemotherapy or with AIDS), C. albicans can also cause disseminated bloodstream infections with mortality rates exceeding 40\% (Wenzel, 1995; Calderone and Fonzi, 2001; Douglas, 2003; Pappas et al., 2004; López-Ribot, 2005).

An important virulence trait of C. albicans is its ability to form biofilms, structured communities of cells several hundred microns thick, that can form on both biotic and abiotic surfaces (Chandra et al., 2001; Douglas, 2002, 2003; Kumamoto, 2002; Ramage et al., 2009; Fox and Nobile, 2012; 
Lohse et al., 2018). When mature, these biofilms contain a mixture of yeast, pseudohyphal, and hyphal cells surrounded by an extracellular matrix (Chandra et al., 2001; Douglas, 2003; Ramage et al., 2009; Fox and Nobile, 2012; Gulati and Nobile, 2016). C. albicans forms biofilms on mucosal surfaces, epithelial cell linings, and on implanted medical devices, such as catheters, dentures, and heart valves (Kojic and Darouiche, 2004; Ramage et al., 2006). Mature C. albicans biofilms also release yeast cells, which can seed new infections elsewhere in the host (Uppuluri et al., 2010, 2018).

Candida albicans biofilms are typically resistant to antifungal drugs at the concentrations that are normally effective against planktonic (free-floating) cells, thus requiring higher drug concentrations, which can lead to host side effects, such as liver and kidney damage (Donlan, 2001; Kojic and Darouiche, 2004; Ramage et al., 2006; Tumbarello et al., 2007, 2012; Lebeaux et al., 2014). Furthermore, C. albicans can also form polymicrobial biofilms with several companion bacterial species (Bamford et al., 2009, 2015; Jarosz et al., 2009; Peleg et al., 2010; Peters and Noverr, 2013; Lindsay and Hogan, 2014; Pammi et al., 2014; Jack et al., 2015), further complicating treatment strategies. These polymicrobial biofilms can, for example, protect their bacterial inhabitants from environmental hazards (e.g., oxygen in the case of anaerobic bacteria) (Fox et al., 2014) and antibiotic treatments (e.g., protecting Staphylococcus aureus from vancomycin) (Harriott and Noverr, 2009, 2010; Kong et al., 2016). The drug-resistant nature of both single species and polymicrobial biofilms frequently makes removal of biofilminfected medical devices the only treatment. However, this recourse is problematic when patients are critically ill or when device removal involves complicated surgical procedures (e.g., heart valve replacement) (Kojic and Darouiche, 2004; Andes et al., 2012; Fox et al., 2015b).

Currently, the three major classes of antifungal drugs used to treat $C$. albicans infections are the polyenes, azoles, and echinocandins (Fox et al., 2015b; Prasad et al., 2016). The polyenes (e.g., amphotericin B) target ergosterol in the fungal cell membrane and are fungicidal against $C$. albicans. The azoles (e.g., fluconazole) inhibit the demethylase enzyme Erg11 from the ergosterol biosynthesis pathway and are fungistatic against C. albicans. Echinocandins (e.g., caspofungin), the most recently developed class of antifungal drugs, inhibit synthesis of the cell wall crosslinking component $\beta$-1,3-glucan and are fungicidal against $C$. albicans. Although novel derivatives within these classes have been introduced over the years, new classes of drugs have not been introduced. The limited size of the existing antifungals, both in terms of the distinct classes and in the number of drugs within several of these classes, creates several problems. As noted above, these classes of drugs typically have reduced effectiveness against biofilms relative to planktonic cells (Donlan, 2001; Kojic and Darouiche, 2004; Ramage et al., 2006; Tumbarello et al., 2007, 2012; Lebeaux et al., 2014). Furthermore, long term exposure to these drugs, especially to members of the azole class, can give rise to antifungal resistance. Although the development of new antifungal agents is clearly called for, several recent in vitro studies have shown that combinations of antifungals with other extant drugs can be effective against C. albicans biofilms (Delattin et al., 2014; De Cremer et al., 2015).

Recently, we demonstrated the importance of several secreted proteases (Saps) for C. albicans biofilm formation (Nobile et al., 2012; Winter et al., 2016). Deletion of Sap5 and Sap6, both of whose expression is upregulated in biofilms (Nobile et al., 2012), reduced biofilm formation in vitro and in vivo (Winter et al., 2016). Previous reports showed that treatment with aspartyl protease inhibitors, a class of drug commonly used to treat HIV patients, reduced the occurrence of oral candidiasis in immunocompromised patients independent of effects of the drug on the immune system through HIV remediation (Cauda et al., 1999; Diz Dios et al., 1999; Cassone et al., 2002). Further work showed that several of the commonly used antiretroviral HIV aspartyl protease inhibitors could inhibit the Saps (Cassone et al., 1999; Gruber et al., 1999b,a; Korting et al., 1999; Pichová et al., 2001; Skrbec and Romeo, 2002; Cenci et al., 2008; Braga-Silva et al., 2010). Exposure to these protease inhibitors also reduced C. albicans adherence to materials commonly used in medical devices and to layers of host cells (Borg-von Zepelin et al., 1999; Bektic et al., 2001; Tsang and Hong, 2009), although the magnitude of the latter effect differs greatly between distinct cell types (Falkensammer et al., 2007). Aspartyl protease inhibitors have also been observed to reduce $C$. albicans-induced tissue damage, proliferation, and virulence in vivo in a rat vaginal model (Cassone et al., 1999; de Bernardis et al., 1999). Finally, one study suggested that aspartyl protease inhibitors and the antifungal agents fluconazole or amphotericin B act synergistically against C. albicans in the planktonic form (Casolari et al., 2004). To date, the studies of aspartyl protease inhibitors with regards to C. albicans emphasized their effects on planktonic cells. The one exception found that exposure to amprenavir, a common HIV antiretroviral protease inhibitor, could reduce C. albicans biofilm formation in vitro (Braga-Silva et al., 2010).

Given the number of protease inhibitors already approved for use in humans, including inhibitors of aspartyl proteases or other classes of proteases, we sought to evaluate the ability of a wide range of protease inhibitors to prevent (either alone or in combination with other antifungals) the formation of C. albicans biofilms or to act against mature biofilms. To evaluate the efficacy of these compounds in this regard, we screened three libraries containing 80 protease inhibitors in both biofilm inhibition and disruption assays. Each protease inhibitor was screened for biofilm efficacy individually and in combination with fluconazole, amphotericin B, or caspofungin. Although few of the protease inhibitors were effective against biofilms on their own, several, especially members of the aspartyl protease inhibitor class, were effective against biofilms when combined with either caspofungin or amphotericin B.

\section{MATERIALS AND METHODS}

\section{Strains and Media}

All assays were performed using SNY425, a SC5314-derived prototrophic a/ $\alpha$ strain (Noble et al., 2010); the sensitivity of this strain to amphotericin B, caspofungin, and fluconazole in 
our assays are reported in Supplementary Table S1 "SN425 Sensitivity". C. albicans cells were cultured as previously described; in brief, cells were recovered from glycerol stocks for two days at $30^{\circ} \mathrm{C}$ on yeast extract peptone dextrose (YEPD) plates $\left(2 \%\right.$ Bacto $^{\mathrm{TM}}$ peptone, $2 \%$ dextrose, $1 \%$ yeast extract, $2 \%$ agar). Overnight cultures were grown approximately $16 \mathrm{~h}$ at $30^{\circ} \mathrm{C}$ in YEPD media (2\% Bacto $^{\mathrm{TM}}$ peptone, $2 \%$ dextrose, $1 \%$ yeast extract). Biofilm assays were performed in RPMI-1640 media (containing L-glutamine and lacking sodium biocarbonate, MP Biomedicals \#0910601) supplemented with $34.5 \mathrm{~g} / \mathrm{L}$ MOPS (Sigma, M3183), adjusted to pH 7.0 with sodium hydroxide, and sterilized with a $0.22 \mu \mathrm{m}$ filter (Lohse et al., 2017; Gulati et al., 2018).

\section{Compound Libraries}

The 53 member SCREEN-WELL ${ }^{\circledR}$ Protease Inhibitor Library ${ }^{1}$ was purchased from Enzo Life Sciences. The two aspartyl protease inhibitor libraries (from which we focused on nine FDA-approved HIV-1 protease inhibitors, the ten macrocycles, and eight linear peptidomimetics) have been previously reported (Clarke et al., 2016). Due to limited quantities of several aspartyl protease inhibitors, a minority of compounds were only screened in one biofilm assay. In these cases, we prioritized the Disruption Biofilm Assay over the Sustained Inhibition Biofilm Assay. Four other compounds from these libraries [one FDA-approved HIV1 protease inhibitor and three linear peptidomimetics (API7-9)] were not used in any assay. A list of compounds tested can be found in Supplementary Table S1.

\section{Biofilm Assays}

The Sustained Inhibition and Disruption Standard Optical Density Biofilm Assays followed previously reported protocols for the 384-well format of biofilm screening assays (Nobile et al., 2014; Fox et al., 2015a; Lohse et al., 2017; Gulati et al., 2018). Compounds and antifungal agents were added during the 90-min adherence and 24-h growth steps of the Sustained Inhibition Biofilm Assay or for the second 24-h growth step of the Disruption Biofilm Assay. In brief, $1 \mu$ of overnight culture was added to $90 \mu \mathrm{l}$ media (or media with drug) in a well (final $\mathrm{OD}_{600}=0.15$, roughly $2 \times 10^{6} \mathrm{cells} / \mathrm{ml}$ ). Plates were then sealed with Breathe-Easy ${ }^{\circledR}$ sealing membranes (Diversified Biotech BEM-1) and shaken at $37^{\circ} \mathrm{C}$ for $90 \mathrm{~min}$ at $350 \mathrm{rpm}$ in an ELMI (DTS-4) incubator. Media was removed, wells were washed with PBS, and fresh media (or media with drug) was added back to wells. Plates were then resealed and shaken for a further $24 \mathrm{~h}$. For the Sustained Inhibition Biofilm Assay, media was removed at this point and the absorbance $\left(\mathrm{OD}_{600}\right)$ was determined on a Tecan Infinite M1000 Pro or a Tecan M200. For the Disruption Biofilm Assays, media was instead removed in groups of 612 wells and fresh media containing the compound of interest was carefully added back to the wells. Plates were then resealed and shaken for an additional $24 \mathrm{~h}$ before removing media and recording absorbance as described above.

${ }^{1} \mathrm{http} / /$ www.enzolifesciences.com/BML-2833/screen-well-protease-inhibitorlibrary/

\section{Standalone Assays}

Compounds were tested at $40 \mu \mathrm{M}$ in both the Sustained Inhibition and Disruption Standard Optical Density Biofilm Assays (Lohse et al., 2017; Gulati et al., 2018). Individual repeats of candidate compounds and DMSO solvent controls were performed. Each plate had groups of control wells spread throughout the plate to minimize position effects. For the SCREEN-WELL ${ }^{\circledR}$ Protease Inhibitor Library, the 53 compounds were screened once in both the Sustained Inhibition Biofilm Assay and the Disruption Biofilm Assay. Promising compounds from these initial screens were then tested a second time in the relevant assay(s). For the two aspartyl protease inhibitor libraries, we initially screened 21 compounds in the Sustained Inhibition Biofilm Assay and 25 compounds in the Disruption Biofilm Assay. Promising compounds from these initial screens were then tested two more times in the relevant assay(s). An additional three repeats were performed for four compounds (atazanavir, indinavir, nelfinavir, tipranavir) in the Disruption Biofilm Assay. For each experimental set of eight wells, significance was evaluated versus all of the control wells from the same plate by performing Welch's $t$-test (two-tailed, assuming unequal variance). In order to correct for the multiple comparisons performed, we then applied the Bonferroni Correction with $\alpha=0.05$. All of the comparisons for a given type of assay were pooled for this multiple comparisons correction step, giving a number of hypotheses, m, of 104 for the Sustained Inhibition Biofilm Assay and of 125 for the Disruption Biofilm Assay (final thresholds $4.81 \times 10^{-4}$ and $4.00 \times 10^{-4}$, respectively). We then determined whether each experimental repeat had an average absorbance of less than the average of the control wells and was significant after the multiple comparisons correction. To be considered a validated hit, a compound had to satisfy both these criteria. Data and statistics for the Standalone Sustained Inhibition and Disruption Optical Density Biofilm Assays are compiled in Supplementary Table S1 "Standalone Inhibition" and "Standalone Disruption". A summary of hits from these assays are included in Supplementary Table S1 "Hit Listing By Type.”

\section{BIC Assays}

We determined the biofilm inhibitory concentration (BIC) of nelfinavir, tipranavir, and TPCK using the 384-well format Sustained Inhibition Standard Optical Density Biofilm Assay (Lohse et al., 2017; Gulati et al., 2018). Both nelfinavir and tipranavir were serially diluted two-fold from a maximum concentration of $200 \mu \mathrm{M}$ to a minimum concentration of $0.1 \mu \mathrm{M}$. TPCK was serially diluted two-fold from a maximum concentration of $512 \mu \mathrm{M}$ to a minimum concentration of $0.06 \mu \mathrm{M}$. Equivalent volumes of DMSO were used as loading controls for the compounds. Statistical testing was performed as described above with the following changes. Significance was evaluated for a given concentration of compound (e.g., $50 \mu \mathrm{M}$ nelfinavir) compared to the equivalent DMSO loading control (e.g., the $50 \mu \mathrm{M}$ loading control). All BIC comparisons were then pooled for multiple comparisons correction, giving a number of hypotheses, $\mathrm{m}$, of 38 ( $\alpha=0.05$, final threshold 
$\left.1.32 \times 10^{-3}\right)$. We then determined whether each concentration of a drug had an average absorbance of less than the average of the relevant control wells and was significant after the multiple comparisons correction. The BIC of a compound was defined as the lowest concentration that met both of these requirements for which all higher concentrations of the same compound also met these requirements. If no concentration met these requirements, the BIC is indicated as greater than the highest concentration tested for that compound. Data and statistics for the BIC Sustained Inhibition Optical Density Biofilm Assay are compiled in Supplementary Table S1 "Inhibition BIC."

\section{Combination Assays}

The combination (candidate compound plus known antifungal agent) Sustained Inhibition and Disruption Biofilm Assays followed the protocols described above with the following modifications. The candidate compounds were included at $12.5 \mu \mathrm{M}$ in both assays except for TPCK, Dec-RVKR$\mathrm{CMK}$, AEBSF.HCl, N-Ethylmaleimide, and acivicin, which were included at $4 \mu \mathrm{M}$, and gliotoxin, which was included at $1 \mu \mathrm{M}$. The Sustained Inhibition Biofilm Assays used $1 \mu \mathrm{g} / \mathrm{mL}$ amphotericin $\mathrm{B}, 0.125 \mu \mathrm{g} / \mathrm{mL}$ caspofungin, or $256 \mu \mathrm{g} / \mathrm{mL}$ fluconazole. The Disruption Biofilm Assays used $2 \mu \mathrm{g} / \mathrm{mL}$ amphotericin B, $0.5 \mu \mathrm{g} / \mathrm{mL}$ caspofungin, or $256 \mu \mathrm{g} / \mathrm{mL}$ fluconazole. The sensitivity of the strain used in this study to amphotericin B, caspofungin, and fluconazole are reported in Supplementary Table S1 "SN425 Sensitivity."

Compounds and two sets of controls were included for all candidate compounds and antifungal agents tested on a given plate. The first set of controls contained the candidate compound, but no antifungal agent, while the second set of controls contained the antifungal agent, but no candidate compound. The concentration of candidate compound or antifungal agent in these control wells was the same as the experimental wells. In general, one set of wells was included for each experimental or control condition on a given plate. Statistical analysis was performed using Welch's $t$-test and the Bonferroni Correction as described above with the following modifications. Each experimental condition was compared to both the relevant antifungal agent and candidate controls (e.g., a nelfinavir plus caspofungin experiment was compared to the nelfinavir-only control and the caspofungin-only control from the same plate). All of the same comparisons for a given assay were pooled for the multiple comparisons correction, giving a number of hypotheses, $\mathrm{m}$, of 213 for both the antifungal agent and candidate comparisons in the Sustained Inhibition Biofilm Assay $\left(\alpha=0.05\right.$, final threshold $\left.2.35 \times 10^{-4}\right)$. The number of hypotheses, $\mathrm{m}$, was 240 for both the antifungal agent and candidate comparisons in the Disruption Biofilm Assay $\left(\alpha=0.05\right.$, final threshold $\left.2.08 \times 10^{-4}\right)$. To be considered a hit, any given experimental condition must have an average absorbance of less than the averages of both sets of relevant control wells and remain significant for both sets of comparisons after the multiple comparisons correction. Data, statistics, and concentrations used for the combination Sustained Inhibition and Disruption Optical Density Biofilm Assays are compiled in Supplementary Table S1 "Combination Inhibition" and "Combination Disruption." A summary of hits from these assays are included in Supplementary Table S1 "Hit Listing By Type."

\section{RESULTS}

\section{Protease Inhibitor Libraries}

We selected three libraries of protease inhibitors to screen for compounds with the abilities to inhibit and/or disrupt C. albicans biofilm formation in vitro. The first library, the SCREEN-WELL ${ }^{\circledR}$ Protease Inhibitor Library (Enzo Life Sciences), contains 53 protease inhibitors effective against several classes of proteases (Supplementary Table S1). The remaining two libraries contain 31 compounds known or predicted to specifically inhibit aspartyl proteases (Clarke et al., 2016), of which we tested 27 in at least one assay. We focused on nine FDA-approved aspartyl protease inhibitors, developed to inhibit HIV-1 protease, ten macrocycles (API12-21), and eight linear peptidomimetics (API1-6, 10, and 11) that were originally synthesized with the goal of identifying new aspartyl protease inhibitors (Clarke et al., 2016).

\section{Standalone Screens}

We screened the three libraries for their abilities to inhibit biofilm formation or to disrupt mature biofilms using the Sustained Inhibition Biofilm Assay and Disruption Biofilm Assay (Lohse et al., 2017; Gulati et al., 2018), respectively. In the Sustained Inhibition Biofilm Assay, compounds were included in media during the $90-\mathrm{m}$ adherence and 24 -h growth steps of the biofilm assay; the compounds were evaluated for their ability to reduce or prevent biofilm formation (Figure 1A). In the Disruption Biofilm Assay, a biofilm was grown for $24 \mathrm{~h}$ before the compound of interest was added. The biofilm was then incubated for an additional $24 \mathrm{~h}$ before determining whether the compound affected the mature biofilm (Figure 1A). In both assays, compounds were tested at a concentration of $40 \mu \mathrm{M}$.

Three of the 53 compounds in the SCREEN-WELL ${ }^{\circledR}$ Protease Inhibitor library, acivicin, gliotoxin, and TPCK, inhibited biofilm formation on their own (Figure 1B, Supplementary Table S1 "Standalone Inhibition"). One of these compounds, gliotoxin, also disrupted mature biofilms on its own (Figure 1C, Supplementary Table S1 "Standalone Disruption"). TPCK irreversibly inhibits chymotrypsin (a serine peptidase) and can also inhibit some cysteine peptidases while gliotoxin inhibits the chymotrypsin-like activity of the $20 \mathrm{~S}$ proteasome. Acivicin, on the other hand, is an inhibitor of gamma-glutamyl transpeptidase, an enzyme that transfers gamma-glutamyl groups from peptide donors to peptide acceptors as well as acting as a hydrolase to remove gamma-glutamyl groups from peptides. None of the 25 aspartyl protease inhibitors tested were able to disrupt mature C. albicans biofilms on their own, and only one of the 22 aspartyl protease inhibitors tested, the HIV-1 protease 


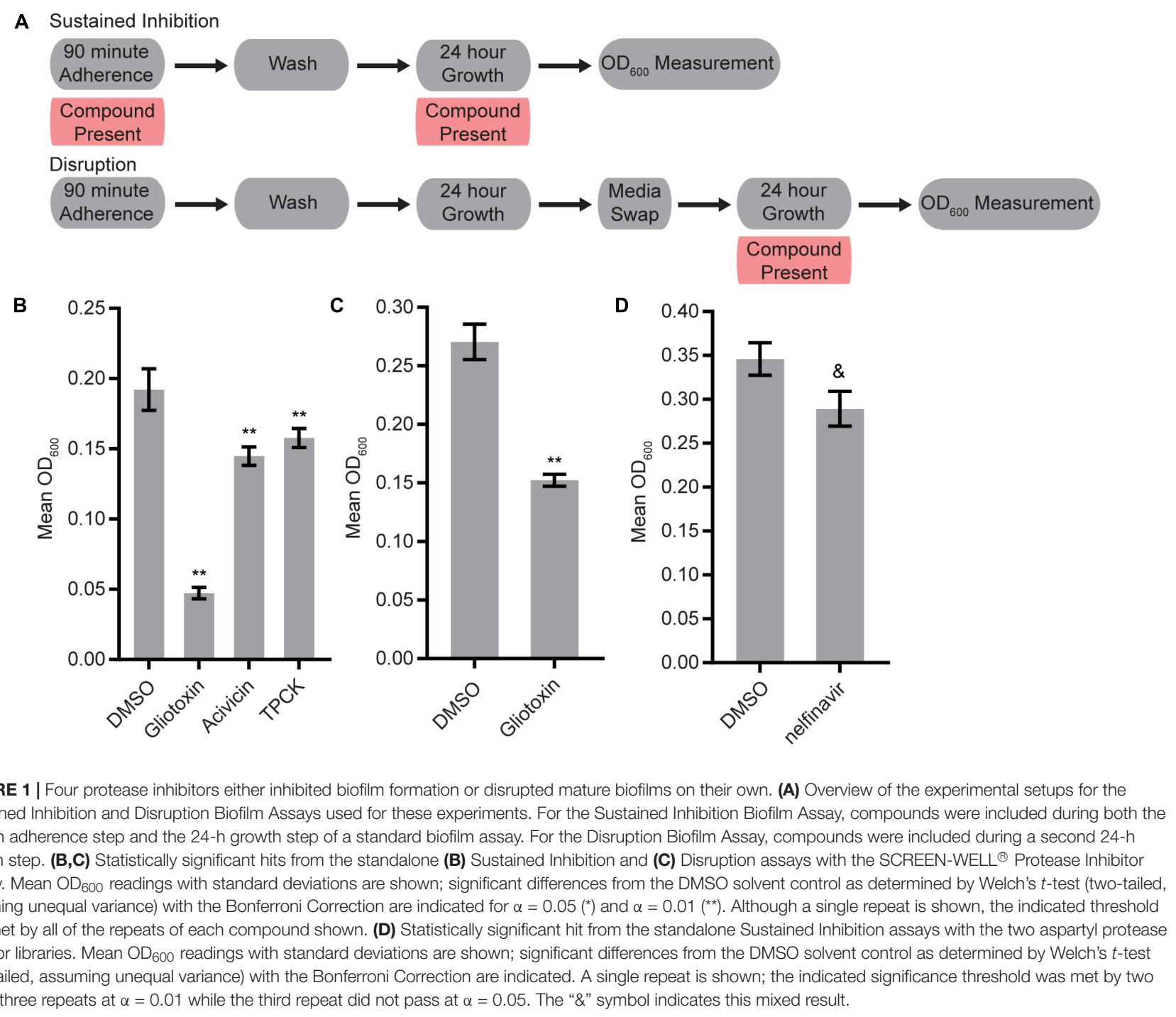

inhibitor nelfinavir, was able to inhibit biofilm formation on its own (BIC $50 \mu \mathrm{M})$ (Figure 1D, Supplementary Table S1 "Standalone Disruption" and "Standalone Inhibition").

\section{Combination Screens}

We tested whether any compounds from the three protease inhibitor libraries could inhibit biofilm formation and/or disrupt mature biofilms in the presence of sub-inhibitory concentrations of amphotericin B, caspofungin, or fluconazole (see methods for concentrations). Five compounds from the SCREEN-WELL ${ }^{\circledR}$ Protease Inhibitor library inhibited biofilm formation in the Sustained Inhibition Biofilm Assay when combined with fluconazole (Figure 2A, Supplementary Table S1 "Combination Inhibition"). We did not observe any synergies with amphotericin B or caspofungin in this assay. Two of these five compounds, gliotoxin and TPCK, were also "hits" in the standalone Sustained Inhibition Biofilm Assay described above. The remaining three compounds, lisinopril, Z-Prolyl-prolinal, and NNGH, were unique to the Sustained Inhibition Biofilm assay for synergies with fluconazole. Lisinopril inhibits the metalloprotease angiotensin-converting enzyme (ACE), NNGH inhibits matrix metalloproteinase 3 (MMP-3), and Z-Prolylprolinal inhibits prolyl endopeptidase (a serine protease). Two compounds from the SCREEN-WELL ${ }^{\circledR}$ Protease Inhibitor library, gliotoxin and Dec-RVKR-CMK, disrupted mature biofilms when combined with an antifungal agent (Figures 2BC, Supplementary Table S1 "Combination Disruption"). Gliotoxin disrupted mature biofilms when combined with fluconazole (Figure 2C, Supplementary Table S1 "Combination Disruption") while Dec-RVKR-CMK disrupted mature biofilms when combined with caspofungin (Figure 2B, Supplementary Table S1 "Combination Disruption"). Dec-RVKR-CMK, also known as furin convertase inhibitor, inhibits the subtilisin (Kex2p-like) proprotein convertase (a type of serine protease).

We next evaluated 17 aspartyl protease inhibitors in the Sustained Inhibition Biofilm Assay and 26 aspartyl protease inhibitors in the Disruption Biofilm Assay in combination with the same three antifungal agents. Seven 


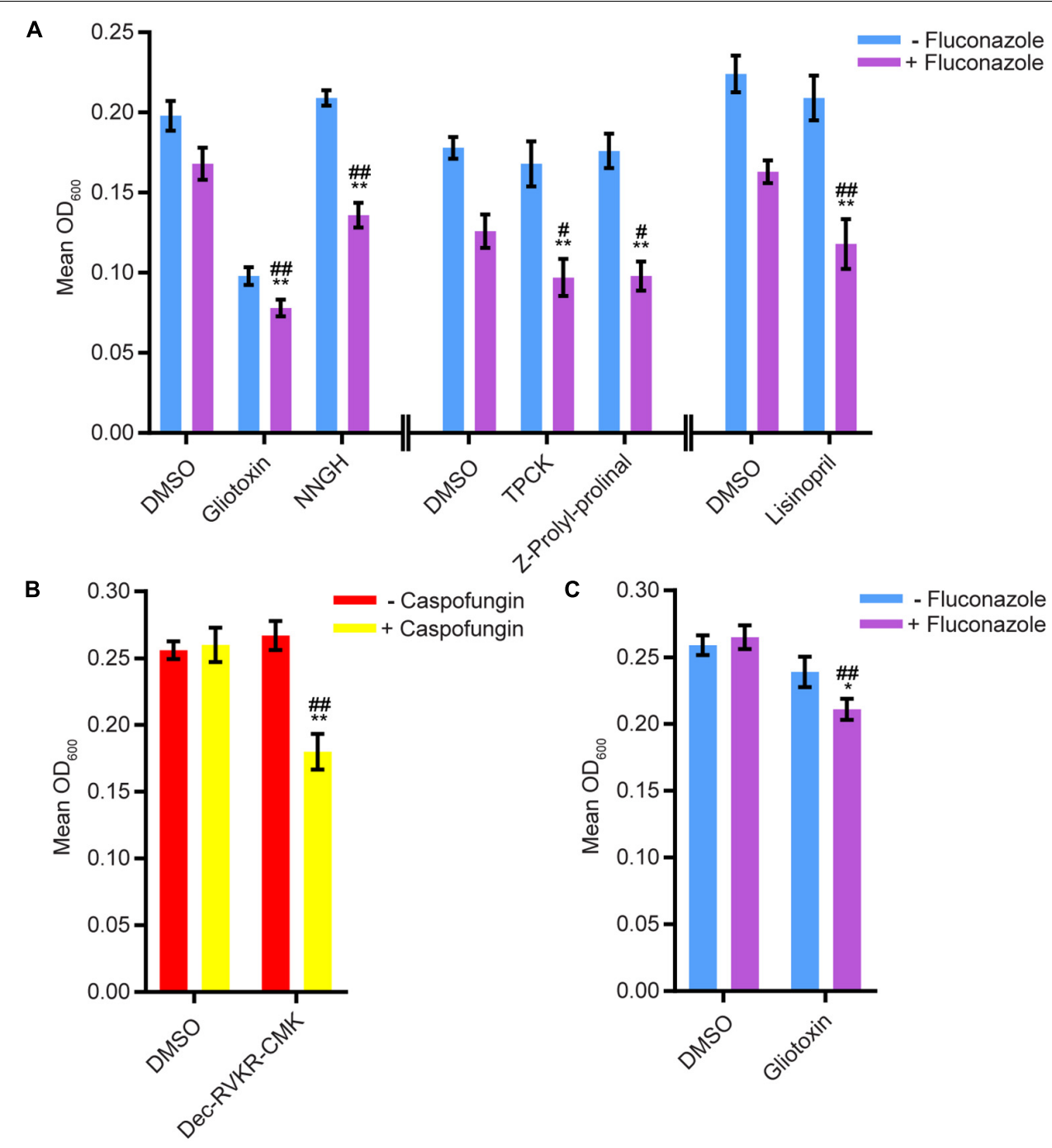

FIGURE 2 | Six compounds from the SCREEN-WELL ${ }^{\circledR}$ Protease Inhibitor Library either inhibited biofilm formation or disrupted mature biofilms in combination with one or more antifungal agents. (A) Statistically significant hits from the combination Sustained Inhibition Biofilm Assays with fluconazole. For each compound, the wells with fluconazole (+fluconazole) are indicated in purple and the wells without fluconazole (-fluconazole) are indicated in blue. (B) Statistically significant hits from the combination Disruption Biofilm Assays with caspofungin. For each compound, the wells with caspofungin (+caspofungin) are indicated in yellow and the wells without caspofungin (-caspofungin) are indicated in red. (C) Statistically significant hits from the combination Disruption Biofilm Assays with fluconazole. For each compound, the wells with fluconazole (+fluconazole) are indicated in purple and the wells without fluconazole (-fluconazole) are indicated in blue. For panels a-c, mean $\mathrm{OD}_{600}$ readings with standard deviations are shown; significant differences from the compound without antifungal agent control (e.g., gliotoxin, - fluconazole), as determined by Welch's $t$-test (two-tailed, assuming unequal variance) with the Bonferroni Correction, are indicated for $\alpha=0.05\left(^{*}\right)$ and $\left.\alpha=0.01{ }^{(\star}\right)$. Significant differences from the antifungal agent without compound control (e.g., DMSO, +fluconazole), as determined by Welch's $t$-test (two-tailed, assuming unequal variance) with the Bonferroni Correction, are indicated for $\alpha=0.05$ (\#) and $\alpha=0.01$ (\#\#). Data from separate plates are separated by two vertical lines on the $x$-axis; the DMSO solvent control is shown for each plate.

aspartyl protease inhibitors (four HIV-1 protease inhibitors and three macrocycles) inhibited biofilm formation when combined with one or more of the antifungal agents (six with caspofungin, five with amphotericin $\mathrm{B}$, and one with fluconazole) (Figure 3, Supplementary Table S1 "Combination Inhibition"). Specifically, lopinavir and API13 inhibited biofilm formation in combination with caspofungin while API19 inhibited biofilm formation in combination with amphotericin B. Ritonavir, saquinavir, and API15 inhibited biofilm formation in combination with caspofungin and amphotericin B while nelfinavir inhibited biofilm formation in combination with all three antifungal agents tested (Figure 3, Supplementary Table S1 "Combination Inhibition"). Nine aspartyl protease inhibitors (the HIV-1 protease inhibitors atazanavir, indinavir, lopinavir, nelfinavir, ritonavir, saquinavir; and the macrocycles API15, API16, API19) disrupted mature biofilms in combination with caspofungin (Figure 4, Supplementary Table S1, "Combination Disruption"). None of the 26 aspartyl protease inhibitors tested disrupted biofilms in the presence of amphotericin B or fluconazole. We were surprised to find compounds that 


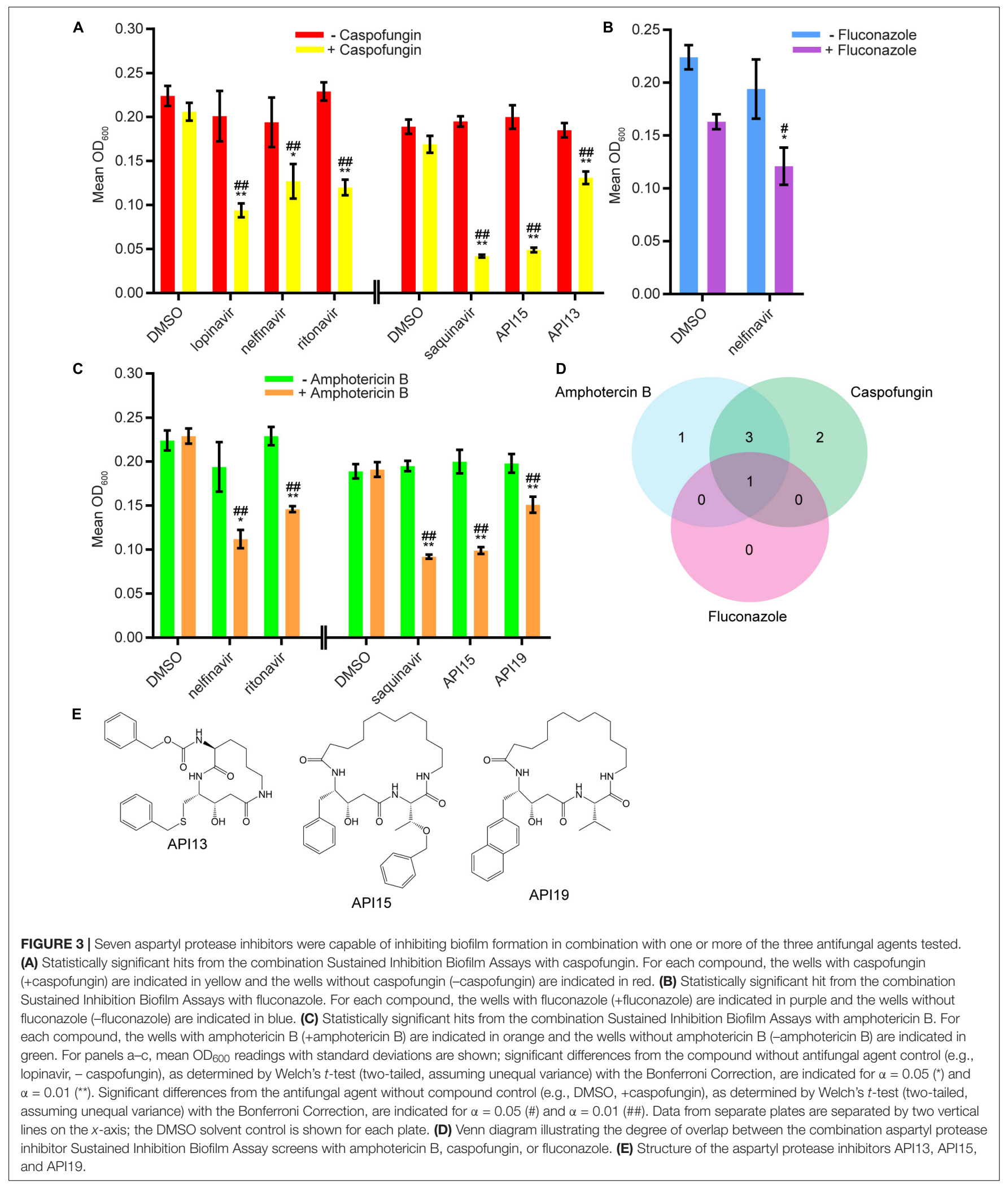

were effective at disrupting mature biofilms, but were not effective at inhibiting biofilm formation, namely atazanavir, indinavir, and API16. We also note that the macrocycle
API19 had a synergistic effect with amphotericin B in the Sustained Inhibition Biofilm Assay but with caspofungin in the Disruption Biofilm Assay. 


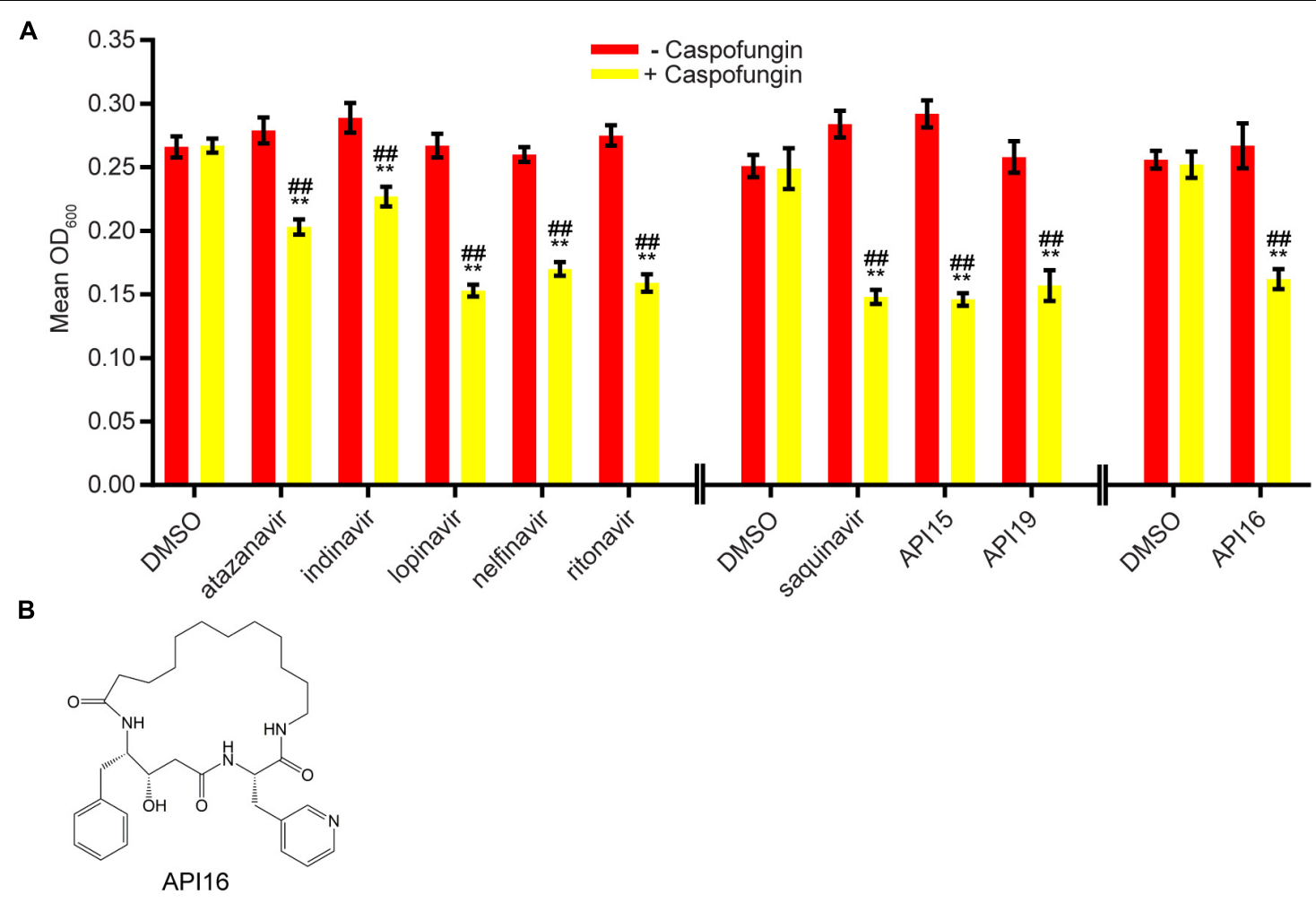

FIGURE 4 | Nine aspartyl protease inhibitors disrupted mature biofilms in combination with the antifungal agent caspofungin. (A) Statistically significant hits from the combination Disruption Biofilm Assays with caspofungin. For each compound, the wells with caspofungin (+caspofungin) are indicated in yellow and the wells without caspofungin (-caspofungin) are indicated in red. Mean $\mathrm{OD}_{600}$ readings with standard deviations are shown; significant differences from the compound without the caspofungin control (e.g., atazanavir, - caspofungin), as determined by Welch's t-test (two-tailed, assuming unequal variance) with the Bonferroni Correction, are indicated for $\alpha=0.05\left(^{\star}\right)$ and $\alpha=0.01\left(^{\star *}\right)$. Significant differences from the caspofungin without compound control (e.g., DMSO, +caspofungin), as determined by Welch's $t$-test (two-tailed, assuming unequal variance) with the Bonferroni Correction, are indicated for $\alpha=0.05$ (\#) and $\alpha=0.01$ (\#\#). Data from separate plates are separated by two vertical lines on the $x$-axis; the DMSO solvent control is shown for each plate. (B) Structure of the aspartyl protease inhibitor API16.

\section{DISCUSSION}

The ability of C. albicans to form biofilms on biotic and abiotic surfaces presents a serious treatment challenge in the clinic as biofilms are typically resistant to all classes of antifungal drugs used to treat planktonic infections. Our results suggest that proteolysis is important for the maintenance of the C. albicans biofilm structure since anti-proteolytic agents contribute to the prevention and disruption of these biofilms. Proteases may play several different roles in C. albicans biofilm formation, an idea supported by the fact that proteases are dynamically expressed throughout the course of $C$. albicans biofilm formation (Nailis et al., 2010; Fox et al., 2015a). For example, Sap5 and Sap6, two secreted aspartyl proteases that are highly upregulated at certain stages of biofilm formation, are known to mediate adhesion of $C$. albicans cells to surfaces and possibly of C. albicans cells to one another (Kumar et al., 2015; Winter et al., 2016). Proteases may also contribute to the breakdown and acquisition of nutrients, the processing of molecules important for biofilm formation (e.g., adhesion molecules), quorum sensing, and/or extracellular matrix production throughout biofilm formation and maintenance. Although the involvement of secreted proteases in biofilm formation is a relatively new concept, there is some precedent for this idea in bacterial biofilms, where extracellular proteases were found to be involved in the processing of adhesion molecules during biofilm formation of Staphylococcus species (Koziel and Potempa, 2013; Paharik et al., 2017; Martínez-García et al., 2018).

In this study, we identify several protease inhibitors from different classes that are effective at preventing biofilm formation and/or at disrupting established biofilms when combined with caspofungin, fluconazole, or amphotericin B, members of the three major antifungal classes used to treat fungal infections in the clinic. Aspartyl protease inhibitors, in particular those that inhibit HIV-1 protease, were the most effective compounds tested when combined with traditional antifungal agents. Combined with the known dependence on Sap5 and Sap6 for biofilm formation (Winter et al., 2016) and previous reports that aspartyl protease inhibitors affect C. albicans in vitro and in vivo (Borgvon Zepelin et al., 1999; Cassone et al., 1999, 2002; Cauda et al., 1999; de Bernardis et al., 1999; Diz Dios et al., 1999; Gruber et al., 1999b,a; Korting et al., 1999; Bektic et al., 2001; Pichová et al., 2001; Skrbec and Romeo, 2002; Cenci et al., 2008; Tsang and Hong, 2009; Braga-Silva et al., 2010), aspartyl protease inhibitors are potentially promising combination treatments for C. albicans biofilm infections which are recalcitrant to single drug 
treatments. We note, however, that we screened fewer inhibitors of other classes of proteases than we did for aspartyl proteases. Despite this bias, we succeeded in identifying several inhibitors of two additional classes of proteases, serine and metalloproteases. It may prove rewarding to conduct additional screens of FDAapproved drugs whose mechanisms rely on the inhibition of other classes of proteases with the goal of repurposing these drugs as novel antifungals.

Perhaps the most unexpected result from this study was the identification of compounds capable of disrupting mature biofilms that were unable to prevent biofilm formation (Figure 5). Unlike the opposite case, where a compound that could prevent biofilm formation might be unable to penetrate a mature biofilm to have an effect, it is not readily apparent how the capacity to disrupt an established biofilm would not also inhibit the formation of a biofilm. Although we do not understand the basis for this result, it demonstrates that compounds that disrupt biofilms are not simply a subset of those that inhibit formation (Figure 5). This observation underscores the importance of screening compounds for their antibiofilm capabilities in both types of assays.

Although we focused on one type of compound, protease inhibitors, this study raises several points to consider when screening for antibiofilm agents. First, consistent with previous reports (Delattin et al., 2014; De Cremer et al., 2015), our results highlight the importance of screening for synergistic interactions, as we detected more hits and hits with stronger effects against biofilms when existing antifungal agents were present along with the compound of interest (Figure 5). Second, our results highlight the importance of screening using biofilms as opposed to planktonic cultures. For example, in our biofilm assays with saquinavir, amphotericin B showed more synergy than fluconazole whereas the opposite relationship was reported for planktonic cultures (Casolari et al., 2004). We also note that we identified compounds that had effects on their own but not in combination with existing antifungal agents, as well as the

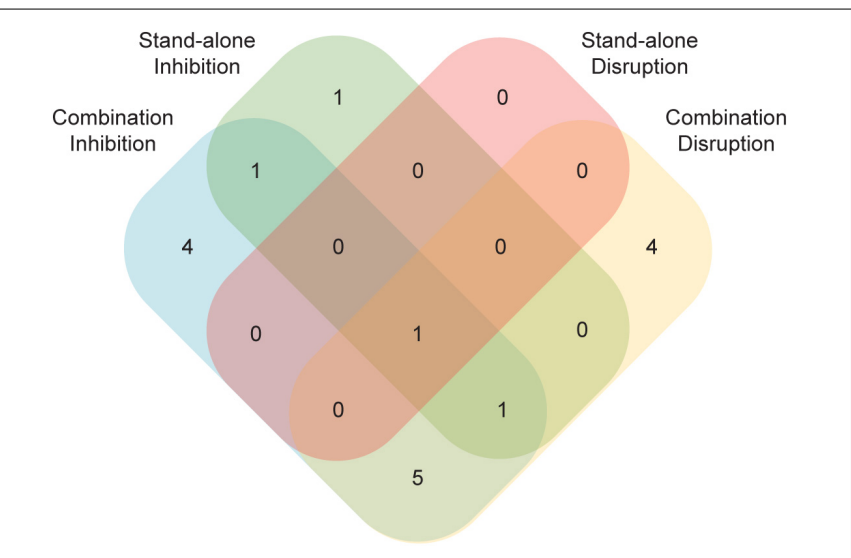

FIGURE 5 | A number of compounds had effects in just a subset of the four biofilm assays. Compounds with an effect in either the standalone or the combination versions of the Sustained Inhibition or Disruption Biofilm Assays are indicated. In total, 17 compounds had an effect in at least one of the four assays. reverse. As such, pursuing multiple assays (e.g., planktonic versus biofilm, standalone compounds versus combinations) maximizes the chance of identifying useful compounds.

Finally, we note that this study was largely inspired by the discovery of the biofilm defects of the sap5 and sap6 single and double mutant strains (Winter et al., 2016). Thus, future compound library screening could be informed by other sets of gene knockouts with biofilm defects; likewise, results from chemical screens could identify genes (and their protein products) required for biofilm formation if the mechanism of action of the chemical compound is known. To further develop the idea of exploiting existing compounds, it should be possible to screen existing C. albicans mutant strain libraries for biofilm defects that arise in the presence of subinhibitory concentrations of traditional antifungal agents. Should biofilm formation by specific classes of mutant strains prove particularly sensitive to traditional antifungal agents, a subsequent combination screen between the traditional antifungal agents and compounds that affect that particular pathway of genes might prove informative.

\section{DATA AVAILABILITY STATEMENT}

All datasets generated for this study are included in the article/Supplementary Material.

\section{AUTHOR CONTRIBUTIONS}

ML, MG, CN, and AJ conceptualized the study. ML and MG worked on the methodology and carried out the investigation. ML contributed to validation, formal analysis, the data curation, and writing the original draft. ML and MG worked on the methodology and carried out the investigation. $\mathrm{CC}, \mathrm{CN}$, and AJ helped with the resources. ML, CC, MG, CN, and AJ reviewed and edited the manuscript. $\mathrm{ML}$ and $\mathrm{CN}$ helped with the visualization. $\mathrm{ML}, \mathrm{CN}$, and $\mathrm{AJ}$ were responsible for the supervision and project administration. $\mathrm{ML}, \mathrm{CC}, \mathrm{CN}$, and $\mathrm{AJ}$ acquired the funding.

\section{FUNDING}

This work was supported by the National Institutes of Health (NIH) grants R43AI131710 (to ML), P50AI150476 (to CC), R01AI083311 (to AJ), and R35GM124594, and R41AI112038 (to $\mathrm{CN})$. This work was also supported by the Kamangar family in the form of an endowed chair (to $\mathrm{CN}$ ). The content is the sole responsibility of the authors and does not represent the views of the funders. The funders had no role in the design of the study; in the collection, analyses, or interpretation of data; in the writing of the manuscript; and in the decision to publish the results.

\section{ACKNOWLEDGMENTS}

We thank Drs. Michael Winter and Starlynn Clarke for advice. This manuscript has been released as a pre-print at bioRxiv (Lohse et al., 2020). 


\section{SUPPLEMENTARY MATERIAL}

The Supplementary Material for this article can be found online at: https://www.frontiersin.org/articles/10.3389/fmicb. 2020.01027/full\#supplementary-material

TABLE S1 | Compiled data and statistics from the standalone and combination Sustained Inhibition and Disruption Optical Density Biofilm Assays as well as the BIC Sustained Inhibition Optical Density Biofilm Assay. For each compound, the

\section{REFERENCES}

Achkar, J. M., and Fries, B. C. (2010). Candida infections of the genitourinary tract. Clin. Microbiol. Rev. 23, 253-273. doi: 10.1128/cmr.00076-09

Andes, D. R., Safdar, N., Baddley, J. W., Playford, G., Reboli, A. C., Rex, J. H., et al. (2012). Impact of treatment strategy on outcomes in patients with candidemia and other forms of invasive candidiasis: a patient-level quantitative review of randomized trials. Clin. Infect. Dis. 54, 1110-1122. doi: 10.1093/cid/cis021

Bamford, C. V., D'Mello, A., Nobbs, A. H., Dutton, L. C., Vickerman, M. M., and Jenkinson, H. F. (2009). Streptococcus gordonii modulates Candida albicans biofilm formation through intergeneric communication. Infect. Immun. 77 , 3696-3704. doi: 10.1128/IAI.00438-09

Bamford, C. V., Nobbs, A. H., Barbour, M. E., Lamont, R. J., and Jenkinson, H. F. (2015). Functional regions of Candida albicans hyphal cell wall protein Als3 that determine interaction with the oral bacterium Streptococcus gordonii. Microbiology 161, 18-29. doi: 10.1099/mic.0.083378-0

Bektic, J., Lell, C. P., Fuchs, A., Stoiber, H., Speth, C., Lass-Flörl, C., et al. (2001). HIV protease inhibitors attenuate adherence of Candida albicans to epithelial cells in vitro. FEMS Immunol. Med. Microbiol. 31, 65-71. doi: 10.1111/j.1574695x.2001.tb01588.x

Borg-von Zepelin, M., Meyer, I., Thomssen, R., Würzner, R., Sanglard, D., Telenti, A., et al. (1999). HIV-protease inhibitors reduce cell adherence of Candida albicans strains by inhibition of yeast secreted aspartic proteases. J. Invest. Dermatol. 113, 747-751. doi: 10.1046/j.1523-1747.1999.00747.x

Braga-Silva, L. A., Mogami, S. S. V., Valle, R. S., Silva-Neto, I. D., and Santos, A. L. S. (2010). Multiple effects of amprenavir against Candida albicans. FEMS Yeast Res. 10, 221-224. doi: 10.1111/j.1567-1364.2009.00595.x

Calderone, R. A., and Fonzi, W. A. (2001). Virulence factors of Candida albicans. Trends Microbiol. 9, 327-335.

Casolari, C., Rossi, T., Baggio, G., Coppi, A., Zandomeneghi, G., Ruberto, A., et al. (2004). Interaction between saquinavir and antimycotic drugs on C. albicans and C. neoformans strains. Pharmacol. Res. 50, 605-610. doi: 10.1016/j.phrs. 2004.06.008

Cassone, A., De Bernardis, F., Torosantucci, A., Tacconelli, E., Tumbarello, M., and Cauda, R. (1999). In vitro and in vivo anticandidal activity of human immunodeficiency virus protease inhibitors. J. Infect. Dis. 180, 448-453. doi: $10.1086 / 314871$

Cassone, A., Tacconelli, E., De Bernardis, F., Tumbarello, M., Torosantucci, A., Chiani, P., et al. (2002). Antiretroviral therapy with protease inhibitors has an early, immune reconstitution-independent beneficial effect on Candida virulence and oral candidiasis in human immunodeficiency virus-infected subjects. J. Infect. Dis. 185, 188-195. doi: 10.1086/338445

Cauda, R., Tacconelli, E., Tumbarello, M., Morace, G., De Bernardis, F., Torosantucci, A., et al. (1999). Role of protease inhibitors in preventing recurrent oral candidosis in patients with HIV infection: a prospective casecontrol study. J. Acquir. Immune Defic. Syndr. 21, 20-25. doi: 10.1097/ 00126334-199905010-00003

Cenci, E., Francisci, D., Belfiori, B., Pierucci, S., Baldelli, F., Bistoni, F., et al. (2008). Tipranavir exhibits different effects on opportunistic pathogenic fungi. J. Infect. 56, 58-64. doi: 10.1016/j.jinf.2007.08.004

Chandra, J., Kuhn, D. M., Mukherjee, P. K., Hoyer, L. L., McCormick, T., and Ghannoum, M. A. (2001). Biofilm formation by the fungal pathogen Candida albicans: development, architecture, and drug resistance. J. Bacteriol. 183, 5385-5394.

Clarke, S. C., Dumesic, P. A., Homer, C. M., O’Donoghue, A. J., La Greca, F., Pallova, L., et al. (2016). Integrated activity and genetic profiling of secreted concentration used, average $\mathrm{OD}_{600}$, average $\mathrm{OD}_{600}$ of relevant control(s), and value(s) for Welch's $t$-test versus the relevant control(s) are provided. Whether the average $\mathrm{OD}_{600}$ was below the average $\mathrm{OD}_{600}$ of the relevant control(s) and whether the difference from the relevant control(s) remains significant following the Bonferroni Correction $(\alpha=0.05)$ are also indicated. The sensitivity of SNY425 to amphotericin B, caspofungin, and fluconazole in planktonic and biofilms assays are also indicated. A list of the 80 compounds from the three protease inhibitor libraries tested in this study and a summary of the hits from the standalone and combination Sustained Inhibition and Disruption Optical Density Biofilm Assays are also included.

peptidases in Cryptococcus neoformans reveals an aspartyl peptidase required for low pH survival and virulence. PLoS Pathog. 12:e1006051. doi: 10.1371/ journal.ppat.1006051

de Bernardis, F., Mondello, F., Scaravelli, G., Pachì, A., Girolamo, A., Agatensi, L., et al. (1999). High aspartyl proteinase production and vaginitis in human immunodeficiency virus-infected women. J. Clin. Microbiol. 37, 1376-1380. doi: $10.1128 / \mathrm{jcm} .37 .5 .1376-1380.1999$

De Cremer, K., Lanckacker, E., Cools, T. L., Bax, M., De Brucker, K., Cos, P., et al. (2015). Artemisinins, new miconazole potentiators resulting in increased activity against Candida albicans biofilms. Antimicrob. Agents Chemother. 59, 421-426. doi: 10.1128/AAC.04229-14

Delattin, N., De Brucker, K., Vandamme, K., Meert, E., Marchand, A., Chaltin, P., et al. (2014). Repurposing as a means to increase the activity of amphotericin $B$ and caspofungin against Candida albicans biofilms. J. Antimicrob. Chemother. 69, 1035-1044. doi: 10.1093/jac/dkt449

Diz Dios, P., Ocampo, A., Miralles, C., Otero, I., Iglesias, I., and Rayo, N. (1999). Frequency of oropharyngeal candidiasis in HIV-infected patients on protease inhibitor therapy. Oral Surg Oral Med. Oral Pathol. Oral Radiol. Endod. 87, 437-441. doi: 10.1016/s1079-2104(99)70242-8

Donlan, R. M. (2001). Biofilm formation: a clinically relevant microbiological process. Clin. Infect. Dis. 33, 1387-1392. doi: 10.1086/322972

Douglas, L. J. (2002). Medical importance of biofilms in Candida infections. Rev. Iberoam. Micol. 19, 139-143.

Douglas, L. J. (2003). Candida biofilms and their role in infection. Trends Microbiol. 11, 30-36. doi: 10.1016/s0966-842x(02)00002-1

Falkensammer, B., Pilz, G., Bektiæ, J., Imwidthaya, P., Jöhrer, K., Speth, C., et al. (2007). Absent reduction by HIV protease inhibitors of Candida albicans adhesion to endothelial cells. Mycoses 50, 172-177. doi: 10.1111/j.1439-0507. 2006.01353.x

Fox, E. P., Bui, C. K., Nett, J. E., Hartooni, N., Mui, M. C., Andes, D. R., et al. (2015a). An expanded regulatory network temporally controls Candida albicans biofilm formation. Mol. Microbiol. 96, 1226-1239. doi: 10.1111/mmi.13002

Fox, E. P., Singh-Babak, S. D., Hartooni, N., and Nobile, C. J. (2015b). "Biofilms and antifungal resistance," in Antifungals: From Genomics to Resistance and the Development of Novel Agents, eds A. T. Coste and P. Vandeputte (Norfolk: Caister Academic Press), 71-90. doi: 10.21775/9781910190012.04

Fox, E. P., Cowley, E. S., Nobile, C. J., Hartooni, N., Newman, D. K., and Johnson, A. D. (2014). Anaerobic bacteria grow within Candida albicans biofilms and induce biofilm formation in suspension cultures. Curr. Biol. 24, 2411-2416. doi: 10.1016/j.cub.2014.08.057

Fox, E. P., and Nobile, C. J. (2012). A sticky situation: untangling the transcriptional network controlling biofilm development in Candida albicans. Transcription 3, 315-322. doi: 10.4161/trns.22281

Ganguly, S., and Mitchell, A. P. (2011). Mucosal biofilms of Candida albicans. Curr. Opin. Microbiol. 14, 380-385. doi: 10.1016/j.mib.2011.06.001

Gruber, A., Berlit, J., Speth, C., Lass-Flörl, C., Kofler, G., Nagl, M., et al. (1999a). Dissimilar attenuation of Candida albicans virulence properties by human immunodeficiency virus type 1 protease inhibitors. Immunobiology 201, 133144. doi: 10.1016/s0171-2985(99)80052-7

Gruber, A., Speth, C., Lukasser-Vogl, E., Zangerle, R., Borg-von Zepelin, M., Dierich, M. P., et al. (1999b). Human immunodeficiency virus type 1 protease inhibitor attenuates Candida albicans virulence properties in vitro. Immunopharmacology 41, 227-234. doi: 10.1016/s0162-3109(99)00035-1

Gulati, M., Lohse, M. B., Ennis, C. L., Gonzalez, R. E., Perry, A. M., Bapat, P., et al. (2018). In vitro culturing and screening of Candida albicans biofilms. Curr. Protoc. Microbiol. 50:e60. doi: 10.1002/cpmc.60 
Gulati, M., and Nobile, C. J. (2016). Candida albicans biofilms: development, regulation, and molecular mechanisms. Microbes Infect. 18, 310-321. doi: 10. 1016/j.micinf.2016.01.002

Harriott, M. M., and Noverr, M. C. (2009). Candida albicans and Staphylococcus aureus form polymicrobial biofilms: effects on antimicrobial resistance. Antimicrob. Agents Chemother. 53, 3914-3922. doi: 10.1128/AAC.00657-09

Harriott, M. M., and Noverr, M. C. (2010). Ability of Candida albicans mutants to induce Staphylococcus aureus vancomycin resistance during polymicrobial biofilm formation. Antimicrob. Agents Chemother. 54, 3746-3755. doi: 10.1128/ AAC.00573-10

Jack, A. A., Daniels, D. E., Jepson, M. A., Vickerman, M., Lamont, R. J., Jenkinson, H. F., et al. (2015). Streptococcus gordonii comCDE (competence) operon modulates biofilm formation with Candida albicans. Microbiology 161, 411421. doi: 10.1099/mic. 0.000010

Jarosz, L. M., Deng, D. M., van der Mei, H. C., Crielaard, W., and Krom, B. P. (2009). Streptococcus mutans competence-stimulating peptide inhibits Candida albicans hypha formation. Eukaryot. Cell 8, 1658-1664. doi: 10.1128/EC. 00070-09

Kennedy, M. J., and Volz, P. A. (1985). Ecology of Candida albicans gut colonization: inhibition of Candida adhesion, colonization, and dissemination from the gastrointestinal tract by bacterial antagonism. Infect. Immun. 49, 654-663. doi: 10.1128/iai.49.3.654-663.1985

Kim, J., and Sudbery, P. (2011). Candida albicans, a major human fungal pathogen. J. Microbiol. 49, 171-177. doi: 10.1007/s12275-011-1064-7

Kojic, E. M., and Darouiche, R. O. (2004). Candida infections of medical devices. Clin. Microbiol. Rev. 17, 255-267. doi: 10.1128/cmr.17.2.255-267. 2004

Kong, E. F., Tsui, C., Kucharíková, S., Andes, D., Van Dijck, P., and JabraRizk, M. A. (2016). Commensal protection of Staphylococcus aureus against antimicrobials by Candida albicans biofilm matrix. mBio 7:e01365-16.

Korting, H. C., Schaller, M., Eder, G., Hamm, G., Böhmer, U., and Hube, B. (1999). Effects of the human immunodeficiency virus (HIV) proteinase inhibitors saquinavir and indinavir on in vitro activities of secreted aspartyl proteinases of Candida albicans isolates from HIV-infected patients. Antimicrob. Agents Chemother. 43, 2038-2042. doi: 10.1128/aac.43.8.2038

Koziel, J., and Potempa, J. (2013). Protease-armed bacteria in the skin. Cell Tissue Res. 351, 325-337. doi: 10.1007/s00441-012-1355-2

Kullberg, B. J., and Oude Lashof, A. M. L. (2002). Epidemiology of opportunistic invasive mycoses. Eur. J. Med. Res. 7, 183-191.

Kumamoto, C. A. (2002). Candida biofilms. Curr. Opin. Microbiol. 5, 608-611.

Kumamoto, C. A. (2011). Inflammation and gastrointestinal Candida colonization. Curr. Opin. Microbiol. 14, 386-391. doi: 10.1016/j.mib.2011. 07.015

Kumar, R., Saraswat, D., Tati, S., and Edgerton, M. (2015). Novel aggregation properties of Candida albicans secreted aspartyl proteinase Sap6 mediate virulence in oral candidiasis. Infect. Immun. 83, 2614-2626. doi: 10.1128/iai. 00282-15

Lebeaux, D., Ghigo, J. M., and Beloin, C. (2014). Biofilm-related infections: bridging the gap between clinical management and fundamental aspects of recalcitrance toward antibiotics. Microbiol. Mol. Biol. Rev. 78, 510-543. doi: 10.1128/mmbr.00013-14

Lindsay, A. K., and Hogan, D. A. (2014). Candida albicans: molecular interactions with Pseudomonas aeruginosa and Staphylococcus aureus. Fungal Biol. Rev. 28, 85-96. doi: 10.1016/j.fbr.2014.10.002

Lohse, M. B., Gulati, M., Arevalo, A. V., Fishburn, A., Johnson, A. D., and Nobile, C. J. (2017). Assessment and optimizations of Candida albicans in vitro biofilm assays. Antimicrob. Agents Chemother. 61:e02749-16.

Lohse, M. B., Gulati, M., Craik, C. S., Johnson, A. D., and Nobile, C. J. (2020). Combination of antifungal drugs and protease inhibitors prevent Candida albicans biofilm formation and disrupt mature biofilms. bioRxiv [Preprint]

Lohse, M. B., Gulati, M., Johnson, A. D., and Nobile, C. J. (2018). Development and regulation of single- and multi-species Candida albicans biofilms. Nat. Rev. Microbiol. 16, 19-31. doi: 10.1038/nrmicro.2017.107

López-Ribot, J. L. (2005). Candida albicans biofilms: more than filamentation. Curr. Biol. 15, R453-R455. doi: 10.1016/j.cub.2005.06.020

Martínez-García, S., Rodríguez-Martínez, S., Cancino-Diaz, M. E., and CancinoDiaz, J. C. (2018). Extracellular proteases of Staphylococcus epidermidis: roles as virulence factors and their participation in biofilm. APMIS 126, 177-185. doi: 10.1111/apm.12805

Nailis, H., Kucharíková, S., Ricicová, M., Van Dijck, P., Deforce, D., Nelis, H., et al. (2010). Real-time PCR expression profiling of genes encoding potential virulence factors in Candida albicans biofilms: identification of modeldependent and -independent gene expression. BMC Microbiol. 10:114. doi: 10.1186/1471-2180-10-114

Nobile, C. J., Fox, E. P., Hartooni, N., Mitchell, K. F., Hnisz, D., Andes, D. R., et al. (2014). A histone deacetylase complex mediates biofilm dispersal and drug resistance in Candida albicans. mBio 5:e1201-14. doi: 10.1128/mBio.012 01-14

Nobile, C. J., Fox, E. P., Nett, J. E., Sorrells, T. R., Mitrovich, Q. M., Hernday, A. D., et al. (2012). A recently evolved transcriptional network controls biofilm development in Candida albicans. Cell 148, 126-138. doi: 10.1016/j.cell.2011. 10.048

Nobile, C. J., and Johnson, A. D. (2015). Candida albicans biofilms and human disease. Annu. Rev. Microbiol. 69, 71-92.

Noble, S. M., French, S., Kohn, L. A., Chen, V., and Johnson, A. D. (2010). Systematic screens of a Candida albicans homozygous deletion library decouple morphogenetic switching and pathogenicity. Nat. Genet. 42, 590-598. doi: 10.1038/ng.605

Paharik, A. E., Kotasinska, M., Both, A., Hoang, T.-M. N., Büttner, H., Roy, P., et al. (2017). The metalloprotease SepA governs processing of accumulationassociated protein and shapes intercellular adhesive surface properties in Staphylococcus epidermidis. Mol. Microbiol. 103, 860-874. doi: 10.1111/mmi. 13594

Pammi, M., Zhong, D., Johnson, Y., Revell, P., and Versalovic, J. (2014). Polymicrobial bloodstream infections in the neonatal intensive care unit are associated with increased mortality: a case-control study. BMC Infect. Dis. 14:390. doi: 10.1186/1471-2334-14-390

Pappas, P. G., Rex, J. H., Sobel, J. D., Filler, S. G., Dismukes, W. E., Walsh, T. J., et al. (2004). Guidelines for treatment of candidiasis. Clin. Infect. Dis. 38, 161-189. doi: 10.1086/380796

Peleg, A. Y., Hogan, D. A., and Mylonakis, E. (2010). Medically important bacterial-fungal interactions. Nat. Rev. Microbiol. 8, 340-349. doi: 10.1038/ nrmicro2313

Peters, B. M., and Noverr, M. C. (2013). Candida albicans-Staphylococcus aureus polymicrobial peritonitis modulates host innate immunity. Infect. Immun. 81, 2178-2189. doi: 10.1128/IAI.00265-13

Pichová, I., Pavlícková, L., Dostál, J., Dolejsí, E., Hrusková-Heidingsfeldová, O., Weber, J., et al. (2001). Secreted aspartic proteases of Candida albicans, Candida tropicalis, Candida parapsilosis and Candida lusitaniae. Inhibition with peptidomimetic inhibitors. Eur. J. Biochem. 268, 2669-2677. doi: 10.1046/j. 1432-1327.2001.02152.x

Prasad, R., Shah, A. H., and Rawal, M. K. (2016). Antifungals: mechanism of action and drug resistance. Adv. Exp. Med. Biol. 892, 327-349. doi: 10.1007/978-3319-25304-6_14

Ramage, G., Martínez, J. P., and López-Ribot, J. L. (2006). Candida biofilms on implanted biomaterials: a clinically significant problem. FEMS Yeast Res. 6, 979-986. doi: 10.1111/j.1567-1364.2006.00117.x

Ramage, G., Mowat, E., Jones, B., Williams, C., and Lopez-Ribot, J. (2009). Our current understanding of fungal biofilms. Crit. Rev. Microbiol. 35, 340-355.

Skrbec, D., and Romeo, D. (2002). Inhibition of Candida albicans secreted aspartic protease by a novel series of peptidomimetics, also active on the HIV-1 protease. Biochem. Biophys. Res. Commun. 297, 1350-1353. doi: 10.1016/s0006-291x(02) 02372-0

Tsang, C. S. P., and Hong, I. (2009). HIV protease inhibitors differentially inhibit adhesion of Candida albicans to acrylic surfaces. Mycoses 53, 488-494. doi: 10.1111/j.1439-0507.2009.01743.x

Tumbarello, M., Fiori, B., Trecarichi, E. M., Posteraro, P., Losito, A. R., De Luca, A., et al. (2012). Risk factors and outcomes of candidemia caused by biofilmforming isolates in a tertiary care hospital. PLoS One 7:e33705. doi: 10.1371/ journal.pone.0033705

Tumbarello, M., Posteraro, B., Trecarichi, E. M., Fiori, B., Rossi, M., Porta, R., et al. (2007). Biofilm production by Candida species and inadequate antifungal therapy as predictors of mortality for patients with candidemia. J. Clin. Microbiol. 45, 1843-1850. doi: 10.1128/jcm.00131-07 
Uppuluri, P., Acosta Zaldívar, M., Anderson, M. Z., Dunn, M. J., Berman, J., Lopez Ribot, J. L., et al. (2018). Candida albicans dispersed cells are developmentally distinct from biofilm and planktonic cells. mBio 9:e01338-18. doi: 10.1128/ mBio.01338-18

Uppuluri, P., Chaturvedi, A. K., Srinivasan, A., Banerjee, M., Ramasubramaniam, A. K., Köhler, J. R., et al. (2010). Dispersion as an important step in the Candida albicans biofilm developmental cycle. PLoS Pathog. 6:e1000828. doi: 10.1371/ journal.ppat.1000828

Wenzel, R. P. (1995). Nosocomial candidemia: risk factors and attributable mortality. Clin. Infect. Dis. 20, 1531-1534. doi: 10.1093/clinids/20.6. 1531

Winter, M. B., Salcedo, E. C., Lohse, M. B., Hartooni, N., Gulati, M., Sanchez, H., et al. (2016). Global identification of biofilm-specific proteolysis in Candida albicans. mBio 7:e01514-16. doi: 10.1128/mBio.015 $14-16$
Conflict of Interest: $\mathrm{CN}$ and $\mathrm{AJ}$ are cofounders of BioSynesis, Inc., a company developing inhibitors and diagnostics of C. albicans biofilms. ML was formerly an employee and currently is a consultant for BioSynesis, Inc. MG was formerly a consultant for BioSynesis, Inc.

The remaining author declares that the research was conducted in the absence of any commercial or financial relationships that could be construed as a potential conflict of interest.

Copyright (c) 2020 Lohse, Gulati, Craik, Johnson and Nobile. This is an open-access article distributed under the terms of the Creative Commons Attribution License (CC BY). The use, distribution or reproduction in other forums is permitted, provided the original author(s) and the copyright owner(s) are credited and that the original publication in this journal is cited, in accordance with accepted academic practice. No use, distribution or reproduction is permitted which does not comply with these terms. 